Research article

Open Access

\title{
Resident macrophages influence stem cell activity in the mammary gland
}

\author{
David E Gyorki ${ }^{1,2}$, Marie-Liesse Asselin-Labat ${ }^{1}$, Nico van Rooijen ${ }^{3}$, Geoffrey J Lindeman ${ }^{1,4,5}$ and \\ Jane $\mathrm{E}$ Visvader ${ }^{1}$
}

\author{
1VBCRC Laboratory, The Walter and Eliza Hall Institute of Medical Research, 1G Royal Parade, Parkville, Vic 3052, Australia \\ 2Department of Medical Biology, The University of Melbourne, Parkville, Vic 3010, Australia \\ 3Department of Molecular Cell Biology, Vrije Universiteit Medical Center, Van der Boechorstraat 7, 1081 BT, Amsterdam, The Netherlands \\ ${ }^{4}$ Department of Medical Oncology, The Royal Melbourne Hospital, Grattan Street, Parkville, Vic 3050, Australia \\ ${ }^{5}$ Department of Medicine, The University of Melbourne, Royal Melbourne Hospital, Parkville, Vic 3050, Australia \\ Corresponding author: Jane E Visvader, visvader@wehi.edu.au
}

Received: 12 Jun 2009 Revisions requested: 7 Jul 2009 Revisions received: 7 Aug 2009 Accepted: 26 Aug 2009 Published: 26 Aug 2009

Breast Cancer Research 2009, 11:R62 (doi:10.1186/bcr2353)

This article is online at: http://breast-cancer-research.com/content/11/4/R62

(c) 2009 Gyorki et al.; licensee BioMed Central Ltd.

This is an open access article distributed under the terms of the Creative Commons Attribution License (http://creativecommons.org/licenses/by/2.0), which permits unrestricted use, distribution, and reproduction in any medium, provided the original work is properly cited.

\begin{abstract}
Introduction Macrophages in the mammary gland are essential for morphogenesis of the ductal epithelial tree and have been implicated in promoting breast tumor metastasis. Although it is well established that macrophages influence normal mammopoiesis, the mammary cell types that these accessory cells influence have not been determined. Here we have explored a role for macrophages in regulating mammary stem cell (MaSC) activity, by assessing the ability of MaSCs to reconstitute a mammary gland in a macrophage-depleted fat pad.
\end{abstract}

Methods Two different in vivo models were used to deplete macrophages from the mouse mammary fat pad, allowing us to examine the effect of macrophage deficiency on the mammary repopulating activity of MaSCs. Both the Csf1oplop mice and clodronate liposome-mediated ablation models entailed transplantation studies using the MaSC-enriched population.
Results We show that mammary repopulating ability is severely compromised when the wild-type MaSC-enriched subpopulation is transplanted into Csf1op/op fat pads. In reciprocal experiments, the MaSC-enriched subpopulation from Csf1oplop glands had reduced regenerative capacity in a wildtype environment. Utilizing an alternative strategy for selective depletion of macrophages from the mammary gland, we demonstrate that co-implantation of the MaSC-enriched subpopulation with clodronate-liposomes leads to a marked decrease in repopulating frequency and outgrowth potential.

Conclusions Our data reveal a key role for mammary gland macrophages in supporting stem/progenitor cell function and suggest that MaSCs require macrophage-derived factors to be fully functional. Macrophages may therefore constitute part of the mammary stem cell niche.

\section{Introduction}

Increasing evidence suggests that macrophages play a role in the normal development of specific organs. In the pubertal mammary gland, macrophages are recruited to the highly mitotic terminal end buds (TEBs) from which ducts elongate and branch to give rise to a mature ductal tree. Macrophages are presumed to play a role in tissue remodeling by engulfing apoptotic epithelial cells during ductal morphogenesis [1,2]. Colony stimulating factor-1 (Csf1; also referred to as macrophage-Csf) is a key growth factor that regulates the proliferation and survival of macrophages [3]. Csf1oplop mice, homozygous for a null mutation in Csf1, exhibit multiple defects and have reduced macrophage numbers in most tissues including the mammary gland. These mice are severely runted, toothless and osteopetrotic, owing to an osteoclast deficiency that impairs bone resorption. The mammary glands of Csf1oplop mice display lower numbers of terminal end buds as well as reduced ductal branching and elongation [4]. During pregnancy, Csf1op/op glands develop precocious alveolar units but fail to switch to the lactational state resulting in impaired lactation [5]. Mammary-specific rescue has shown that the mammary gland phenotype is not due to secondary 
effects induced by a systemic Csf1 deficiency [6]. Targeted ablation of the receptor for Csf1 (Csf1r or c-fms), recapitulated all the phenotypes observed in Csf1oplop mice, although the receptor-null mice exhibited slightly more severe defects [7].

The nature of the mammary stem cell niche is yet to be defined but candidate cell types that may constitute the niche include epithelial cells, macrophages and eosinophils, among other cells in the mammary stroma (fibroblasts and adipocytes). One mechanism that may underlie the requirement of macrophages for normal mammopoiesis could be their interaction with mammary stem cells (MaSCs) $[8,9]$. To explore this possibility, we have utilized two in vivo approaches to determine whether macrophages are required for the function of MaSCs. We report that stem/progenitor cell activity is markedly attenuated by depletion of macrophages from the mammary gland, indicating that macrophages may influence stem cell function.

\section{Materials and methods Mouse strains}

Csf1oplop mice were a kind gift from Dr Robin Anderson (Peter MacCallum Cancer Centre, Melbourne, Australia) and were on a pure BALB/c background. These mice were housed in a clean facility to optimise the viability of the homozygous mutant mice. Rosa26 mice were on a pure FVB/N background. All experiments were approved by the WEHI Animal Ethics Committee, and the care of animals was in accordance with institutional guidelines.

\section{Mammary stem cell preparation and cell culture}

A single cell suspension of mammary cells was prepared from freshly harvested mammary glands and sorted by flow cytometry as previously described [8]. For transplantation assays, freshly harvested cells from the LinCD29hiCD24+ fraction were transplanted in a volume of $10 \mu$ into the de-epithelialized fourth mammary fat pads of three week-old syngeneic mice. Limiting dilution and statistical analyses were carried out as described by Shackleton et al [8]. For experiments using liposomes, the injection solution contained approximately 42 $\mu \mathrm{g}$ of clodronate liposomes per injection volume. Wholemount and histology analyses, as well as staining of mammary glands for LacZ activity using X-Gal (Sigma), were performed as described [8]. Clodronate liposomes were synthesized as previously described [10].

For in vitro cell culture assays, 100 freshly sorted epithelial cells $\left(56\right.$ cells $/ \mathrm{cm}^{2}$ ) were cultured for one week on 25,000 irradiated NIH-3T3 fibroblasts $\left(14,000\right.$ cells $\left./ \mathrm{cm}^{2}\right)$ in 24 -well culture dishes as described [8]. Colony size was measured using ImageJ [11].

\section{Results}

To evaluate whether the impaired ductal morphogenesis that occurs in Csf1oplop mice (Figure 1a) reflects a perturbation in
MaSC activity, freshly sorted CD29hiCD24+ mammary epithelial cells were isolated from BALB/c Csf1op/op and wild-type glands. They were then transplanted into the de-epithelialized fat pads of three-week-old BALB/c mice at limiting dilution. The CD29hiCD24+ subpopulation has previously been shown to be enriched for MaSCs but also contains mature myoepithelial cells and possibly basal progenitor cells [8]. Cells were purified from 12 week-old Csf1op/op mice and eight-week-old wild-type littermates, in order to compensate for the recognised delay in filling of the fat pad that occurs in Csf1oplop mutant mice [4]. The glands were harvested for wholemount analysis eight weeks post-transplantation.

Limiting dilution analysis of four independent experiments revealed that the stem cell frequency was reduced in Csf1opl op mice. The mammary repopulating frequency was 1 in 261 (95\% confidence interval $(\mathrm{Cl})=1 / 166$ to $1 / 408$ ) versus 1 in $97(95 \% \mathrm{Cl}=1 / 66$ to $1 / 141)$ for Csf1op/op and wild-type mammary glands, respectively $(P<0.001$; Table 1$)$. Estimation of the absolute number of mammary repopulating cells revealed an 18-fold decrease in mutant glands (mean of 702 versus 40 cells per gland in wild-type and Csf1op/opmice, respectively). In addition to the decrease in repopulating frequency by CD29hiCD24+ cells in the Csf1oplop group, only 5 of the 45 transplanted fat pads ( $11 \pm 4 \%$; mean \pm standard error of the mean (SEM)) yielded outgrowths filling more than $50 \%$ of the fat pad, whereas 22 of the 45 transplanted fat pads (49 $\pm 4 \%$ ) were filled by outgrowths from wild-type stem cells. Thus, despite being transplanted into a normal microenvironment, the MaSC-enriched population from Csf1oplop mice did not give rise to fully developed mammary outgrowths (Figure $1 \mathrm{~b}$ ), indicating that the function of mammary stem/progenitor cells had been attenuated in the macrophage-depleted environment.

Consistent with the transplantation data, in vitro colony forming assays on fibroblast feeder layers revealed a two-fold reduction in the clonogenic potential of CD29hiCD24+ cells from Csf1oplop mice relative to those from control mice (Figure 1c). The absolute numbers of clonogenic cells in the MaSCenriched population per mammary gland from either wild-type or Csf1op/opmice were estimated to be 14,666 \pm 1530 and $1306 \pm 348$, respectively (mean \pm SEM, $n=3$ ). Notably, a 2.4-fold decrease in colony size was also apparent on comparison of colonies derived from Csf1op/op CD29hiCD24+ cells relative to those from wild-type mice. The addition of F4/80positive mouse mammary macrophages to the clonogenic assays had no discernible effect on colony size or morphology, but may reflect inadequate culture conditions for macrophages in the epithelial cell assays (data not shown).

To investigate a potential role for macrophages in the mammary stem cell microenvironment, the wild-type MaSCenriched population was next transplanted into the macrophage-deficient fat pads of Csf1oplop mice. Two hundred 
(a)
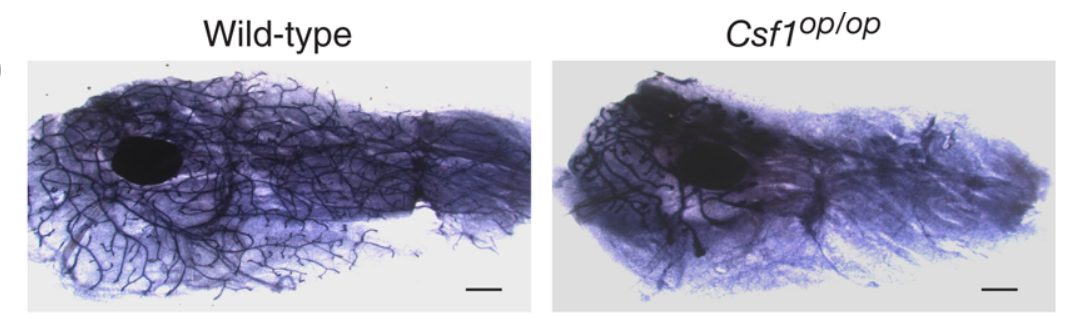

(b)
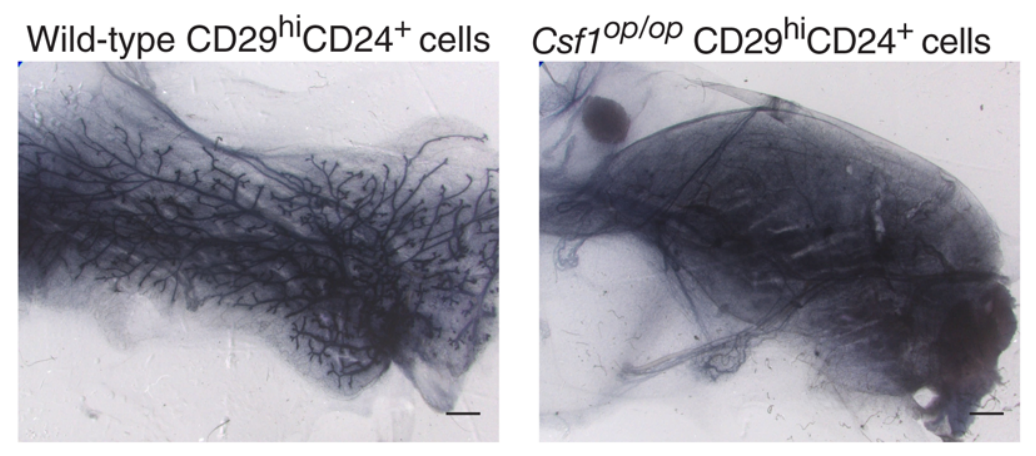

(c)

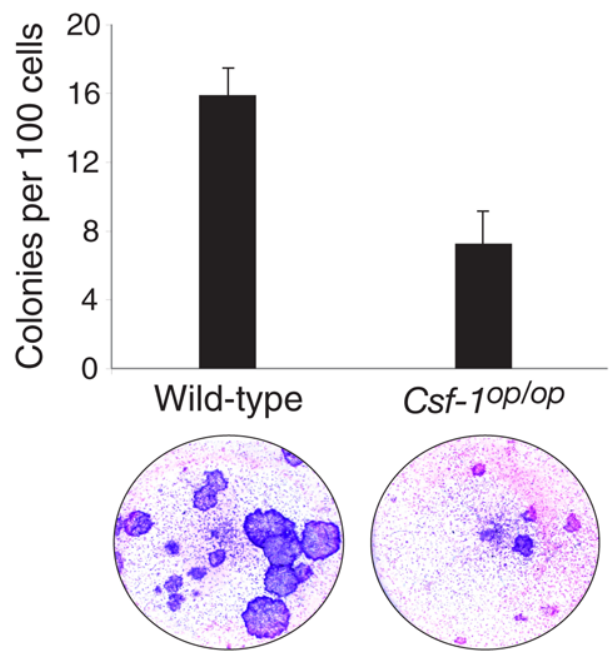

The MaSC-enriched population from Csf1op/op mice yields smaller outgrowths in vivo and has lower clonogenicity in vitro. (a) Wholemounts of fourth mammary glands from eight-week-old wild-type and Csf1op/op mice showing delayed filling of the fat pad and persistence of terminal end buds in mutant animals. (b) Wholemounts of outgrowths following transplantation of CD29hiCD $24^{+}$cells from wild-type or Csf1op/op mammary glands into the cleared fat pads of syngeneic wild-type recipients. (c) CD29hiCD $24^{+}$cells from Csf1op/op mammary glands exhibit lower clonogenicity and colony size than those from control glands. Histogram (upper panel) showing colony forming capacity of $100 \mathrm{CD} 29^{\text {hiCD}} 24^{+}$cells for each genotype. Error bars show standard error of the mean for three independent experiments. Lower panel shows cultures of CD29hiCD24+ cells grown on fibroblast feeder layers. Scale bars $=1 \mathrm{~mm}$. Csf1 = colony stimulating factor $1 ; \mathrm{MaSC}=$ mammary stem cell.

CD29hiCD24+ cells isolated from the mammary glands of young wild-type female mice (BALB/c) were transplanted into either three-week-old wild-type or six-week-old Csf1op/op fat pads that had been cleared of endogenous epithelium. Older mutant recipient mice were used to compensate for the marked developmental delay that occurs in Csf1op/op mice. Substantial outgrowths were observed in wild-type recipients (18 of 24), whereas only a single small outgrowth (1 of 18) comprising two ducts was evident in Csf1op/op recipients $(P<$
0.00001 ; Figures $2 \mathrm{a}, \mathrm{b})$. These data suggest that mammary repopulating cells require the presence of resident macrophages in the mouse mammary gland.

To further investigate the effect of macrophage depletion from the mammary fat pad on stem cell activity, an alternative model of macrophage deficiency was sought. Clodronate is a bisphosphonate that can be delivered in a targeted manner when packaged into liposomes [10]. The liposomes are non- 
Limiting dilution analysis of wild-type or Csf1op/op CD29hiCD24+ cells transplanted into cleared mammary fat pads

\begin{tabular}{|c|c|c|}
\hline \multirow[t]{2}{*}{ Number of CD24+CD29hi cells injected per fat pad } & \multicolumn{2}{|c|}{ Number of positive outgrowths* } \\
\hline & Wild-type & Csf1op/op \\
\hline 60 & $3 / 4$ & $0 / 4$ \\
\hline 100 & $7 / 11$ & $5 / 11$ \\
\hline 120 & $2 / 4$ & $1 / 4$ \\
\hline 200 & $20 / 22$ & $12 / 22$ \\
\hline 240 & $3 / 4$ & $2 / 4$ \\
\hline $\begin{array}{l}\text { Repopulating frequency } \\
\text { (95\% confidence interval) }\end{array}$ & $\begin{array}{c}1 / 97 \\
(1 / 66 \text { to } 1 / 141)\end{array}$ & $\begin{array}{c}1 / 261 \\
(1 / 166 \text { to } 1 / 408)\end{array}$ \\
\hline
\end{tabular}

Limiting dilution analysis of the repopulating frequency of mammary stem cell (MaSC)-enriched cells from either wild-type or Csf1op/op glands transplanted into wild-type recipients. Cells were injected into the cleared mammary fat pads of three-week-old syngeneic recipients. Results are pooled from four independent experiments $(P=0.00065)$. *Shown as number of outgrowths per number of injected fat pads. Csf1 = colony stimulating factor 1 .

toxic until ingestion by macrophages, in which they are broken down by liposomal phospholipases to reveal the drug. Sufficient clodronate uptake by macrophages causes cell death by apoptosis. In co-transplantation experiments, CD29hiCD24+ cells (200 cells) from the mammary glands of Rosa26 mice were coinjected into the cleared mammary fat pads with either clodronate-containing or saline-containing (control) liposomes. Six weeks after transplantation, the glands were harvested and the outgrowths analysed by staining for LacZ activity (see Materials and methods). Three independent experiments revealed outgrowths in 22 of 28 (77 $\pm 5 \%$ ) fat pads coinjected with saline liposomes compared with only 4 of $28(18 \pm 10 \%)$ fat pads co-injected with clodronate-containing liposomes $(P<0.000001)$. Of the four outgrowths evident in the clodronate group, only one branching outgrowth was observed, while the remaining three were very rudimentary structures (Figure 2c).

\section{Discussion}

Our data reveal that mammary macrophages contribute to normal stem/progenitor cell function in the developing mouse mammary gland. In a macrophage-deficient environment, the MaSC-enriched subpopulation had a markedly diminished capacity to reconstitute mammary outgrowths. The role of macrophages in this organ, however, appears to be supportive rather than integral to MaSC function, because these mice harbor some MaSCs and limited development can occur in Csf1oplop mice. Interestingly, even in a macrophage-replete system, the function of MaSCs from Csf1oplop glands was compromised. Indeed, both the absolute number and repopulating capability of MaSCs appear to have been altered by the macrophage-deficient environment, possibly reflecting downregulation of growth factor-mediated signal transduction pathways required for stem cell function.
Here we demonstrate the efficacy of clodronate-containing liposomes for macrophage depletion in the mammary gland. Clodronate liposomes have been successfully utilized for macrophage depletion from liver, spleen, lung, lymph nodes, joints, peritoneal cavity, and testis [10]. Local depletion of resident macrophages from the developing mammary gland by clodronate liposomes led to a marked decrease in repopulation by the MaSC-enriched fraction. The depletion of macrophages is likely to be temporary as the liposomes are phagocytosed within the first few days and macrophages presumably return to normal levels by the time of tissue harvest at six weeks posttransplantation. Therefore, mammary macrophages appear to be required for the early stages of repopulation by MaSCs and their progeny.

It is tempting to speculate that mammary macrophages constitute part of the normal MaSC microenvironment and facilitate the maintenance and/or proliferation of stem cells. Gene expression analysis has shown that the MaSC-enriched subpopulation expresses significantly higher levels of Csf1 (approximately five-fold by quantitiative RT-PCR) relative to the luminal epithelial subset. Only macrophages in the mammary gland appear to express the receptor (c-fms or Csf1R) for the Csf1 ligand under normal physiologic conditions, based on immunostaining for the receptor [4] as well as expression in cfms reporter mice (data not shown). Csf1 may therefore be a chemoattractant for macrophages to the niche in which MaSCs are thought to reside in a basal location. Consistent with this notion, macrophages can be found around the cap cell region of TEBs [4], which has been proposed to be enriched for stem cells [12]. The factors released by macrophages that support stem cell activity are yet to be determined but may include macrophage-secreted cytokines such as CXCL12. Evidence for the direct participation of macrophages in the MaSC niche should eventually be possible through the prospective identification of a highly specific 
(a)
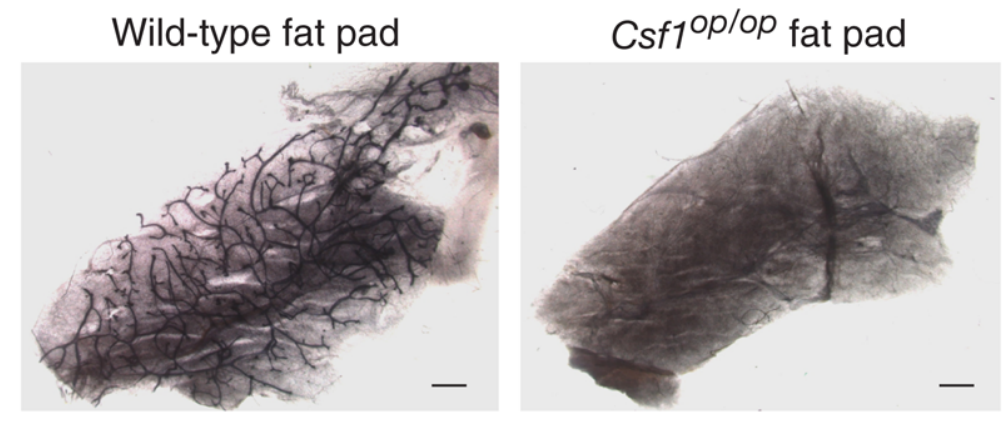

(b)

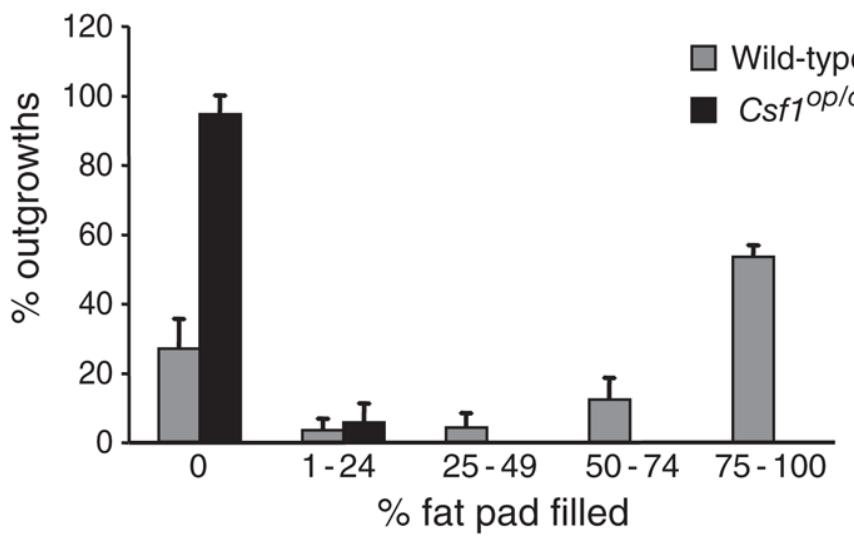

(c)
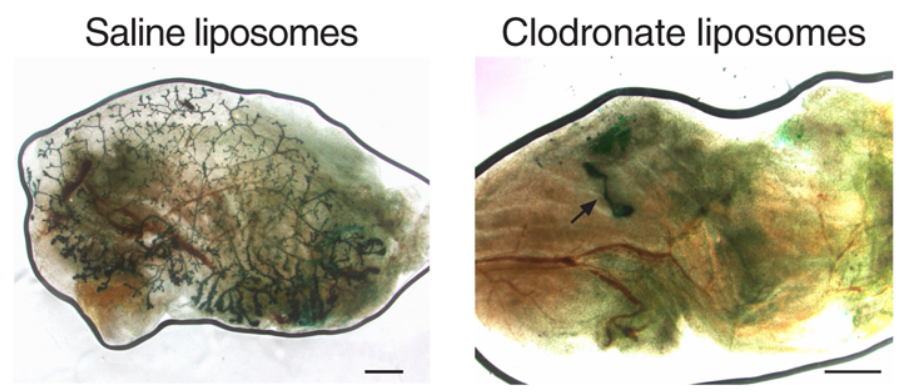

Wild-type mammary stem cells are unable to efficiently repopulate macrophage-deficient fat pads. (a) Wild-type CD29hiCD24+ cells transplanted into Csf1 10 op fat pads resulted in only a single small outgrowth whereas those transplanted into wild-type recipients resulted in extensive filling of the fat pad, all harvested at eight weeks post-transplantation. (b) Histogram showing percent outgrowth and the extent of fat pad filling following transplantation of normal CD29hiCD24+ cells into wild-type or Csf1op/op fat pads. Error bars represent standard error of the mean for three independent experiments. (c) Clodronate liposomes inhibit mammary stem cell function. CD29hiCD24+ cells (200) from Rosa26 mice were co-transplanted with liposomes containing either saline or clodronate into the cleared mammary fat pads of syngeneic wild-type female mice. The cells co-transplanted with clodronate liposomes resulted in no outgrowths or rudimentary structures, an example of which is shown. Scale bars $=1 \mathrm{~mm}$. Csf $1=$ colony stimulating factor 1 .

MaSC marker. The finding that the colonic niche was located at the base of the crypts allowed the localization of macrophages to this region, while electron microscopy identified a direct physical relation between colonic epithelial progenitor cells and macrophages [13].

\section{Conclusions}

Our data demonstrate that depletion of mammary gland macrophages either by chemical ablation or the use of Csf-1op/op mutant mice alters mammary stem/progenitor cell activity, reflected in a substantially reduced repopulating frequency. The diminished outgrowth potential indicates a continued requirement for normal macrophages during ductal morphogenesis and may be mediated by effects on the putative basal progenitor cell. Overall, these findings indicate that macrophages play a role in supporting normal mammary stem cell function in vivo and suggest that they may constitute part of the normal MaSC microenvironment. 


\section{Competing interests}

The authors declare that they have no competing interests.

\section{Authors' contributions}

DG contributed to the conception and design, collection and assembly of data, data analysis and interpretation, manuscript writing. MA contributed to the collection and assembly of data. NvR provided the liposomal reagents. GJL contributed to the study conception and design, provision of study materials, data analysis and interpretation. JEV contributed to the conception and design, provision of study materials, data analysis and interpretation and manuscript writing. All authors read and approved the final manuscript.

\section{Acknowledgements}

We are grateful to S. Holroyd and K. Haunholter for expert technical assistance, to K. Stoev and K. Johnson for excellent animal husbandry, and S. Mihajlovic and E. Tsui for expert assistance with histology. R. Anderson kindly provided the BALB/c Csf1op/op mice. We thank John Hamilton for discussions. This work was supported by the Victorian Breast Cancer Research Consortium, National Health and Medical Research Council (NHMRC, Australia). D. Gyorki received support from the National Health and Medical Research Council, Royal Australasian College of Surgeons and the National Breast Cancer Foundation. M-L Asselin-Labat was the recipient of an ARC Fellowship (Australia).

\section{References}

1. Gouon-Evans V, Lin EY, Pollard JW: Requirement of macrophages and eosinophils and their cytokines/chemokines for mammary gland development. Breast Cancer Res 2002, 4:155-164.

2. Schwertfeger KL, Rosen JM, Cohen DA: Mammary gland macrophages: pleiotropic functions in mammary development. $J$ Mammary Gland Biol Neoplasia 2006, 11:229-238.

3. Pixley FJ, Stanley ER: CSF-1 regulation of the wandering macrophage: complexity in action. Trends Cell Biol 2004, 14:628-638.

4. Gouon-Evans V, Rothenberg ME, Pollard JW: Postnatal mammary gland development requires macrophages and eosinophils. Development 2000, 127:2269-2282.

5. Pollard JW, Hennighausen L: Colony stimulating factor 1 is required for mammary gland development during pregnancy. Proc Natl Acad Sci USA 1994, 91:9312-9316.

6. Van Nguyen A, Pollard JW: Colony stimulating factor-1 is required to recruit macrophages into the mammary gland to facilitate mammary ductal outgrowth. Dev Biol 2002, 247:11-25.

7. Dai XM, Ryan GR, Hapel AJ, Dominguez MG, Russell RG, Kapp S, Sylvestre V, Stanley ER: Targeted disruption of the mouse colony-stimulating factor 1 receptor gene results in osteopetrosis, mononuclear phagocyte deficiency, increased primitive progenitor cell frequencies, and reproductive defects. Blood 2002, 99:111-120.

8. Shackleton M, Vaillant F, Simpson KJ, Stingl J, Smyth GK, AsselinLabat ML, Wu L, Lindeman GJ, Visvader JE: Generation of a functional mammary gland from a single stem cell. Nature 2006, 439:84-88.

9. Stingl J, Eirew P, Ricketson I, Shackleton M, Vaillant F, Choi D, Li $\mathrm{HI}$, Eaves CJ: Purification and unique properties of mammary epithelial stem cells. Nature 2006, 439:993-997.

10. Van Rooijen N, Sanders A: Liposome mediated depletion of macrophages: mechanism of action, preparation of liposomes and applications. J Immunol Methods 1994, 174:83-93.

11. Image] [http://rsb.info.nih.gov/ij/]

12. Kenney NJ, Smith GH, Lawrence E, Barrett JC, Salomon DS: Identification of stem cell units in the terminal end bud and duct of the mouse mammary gland. J Biomed Biotechnol 2001, $1: 133-143$
13. Pull SL, Doherty JM, Mills JC, Gordon JI, Stappenbeck TS: Activated macrophages are an adaptive element of the colonic epithelial progenitor niche necessary for regenerative responses to injury. Proc Natl Acad Sci USA 2005, 102:99-104. 


\section{University Library}

\section{- M M N E R VA A gateway to Melbourne's research publications}

Minerva Access is the Institutional Repository of The University of Melbourne

Author/s:

Gyorki, DE;Asselin-Labat, M-L;van Rooijen, N;Lindeman, GJ;Visvader, JE

Title:

Resident macrophages influence stem cell activity in the mammary gland

Date:

2009-01-01

Citation:

Gyorki, D. E., Asselin-Labat, M. -L., van Rooijen, N., Lindeman, G. J. \& Visvader, J. E.

(2009). Resident macrophages influence stem cell activity in the mammary gland. BREAST

CANCER RESEARCH, 11 (4), https://doi.org/10.1186/bcr2353.

Persistent Link:

http://hdl.handle.net/11343/263756

License:

CC BY 